\title{
Large-Scale Extended Granger Causality for Classification of Marijuana Users From Functional MRI
}

\author{
M. Ali Vosoughi ${ }^{\mathrm{a}}$ and Axel Wismüller ${ }^{\mathrm{a}, \mathrm{b}, \mathrm{c}, \mathrm{d}}$ \\ ${ }^{a}$ Department of Electrical and Computer Engineering, University of Rochester, NY, USA \\ ${ }^{\mathrm{b}}$ Department of Imaging Sciences, University of Rochester, NY, USA \\ ${ }^{c}$ Department of Biomedical Engineering, University of Rochester, NY, USA \\ ${ }^{\mathrm{d}}$ Faculty of Medicine and Institute of Clinical Radiology, Ludwig Maximilian University, \\ Munich, Germany
}

\begin{abstract}
It has been shown in the literature that marijuana use is associated with changes in brain network connectivity. We propose large-scale Extended Granger Causality (lsXGC) and investigate whether it can capture such changes using resting-state fMRI. This method combines dimension reduction with source time-series augmentation and uses predictive time-series modeling for estimating directed causal relationships among fMRI time-series. It is a multivariate approach, since it is capable of identifying the interdependence of time-series in the presence of all other time-series of the underlying dynamic system. Here, we investigate whether this model can serve as a biomarker for classifying marijuana users from typical controls using 126 adult subjects with a childhood diagnosis of ADHD form the Addiction Connectome Preprocessed Initiative (ACPI) database. We use brain connections estimated by lsXGC as features for classification. After feature extraction, we perform feature selection by Kendall's-tau rank correlation coefficient followed by classification using a support vector machine. As a reference method, we compare our results with cross-correlation, which is typically used in the literature as a standard measure of functional connectivity. Within a cross-validation scheme of 100 different training/test $(90 \% / 10 \%)$ data splits, we obtain a mean accuracy range of $[0.714,0.985]$ and a mean Area Under the receiver operating characteristic Curve (AUC) range of [0.779, 0.999] across all tested numbers of features for lsXGC, which is significantly better than results obtained with cross-correlation, namely mean accuracy of $[0.728,0.912]$ and mean AUC of $[0.825,0.969]$. Our results suggest the applicability of lsXGC as a potential biomarker for marijuana use.
\end{abstract}

Further author information: (Send correspondence to Ali Vosoughi)

Ali Vosoughi: E-mail: mvosough@ur.rochester.edu 
Keywords: Resting-state fMRI, Large-Scale Extended Granger Causality, functional connectivity, machine learning, support vector machine, Attention Deficit Hyperactivity Disorder (ADHD), effect of marijuana use

\section{INTRODUCTION}

Marijuana has been used as an illegal drug for decades. Despite many concerns about the adverse effects of repeated marijuana abuse on brain functionalities and cognition, considerably few studies have been conducted to investigate the effects of repeated marijuana use on the brain [1]. Currently, the diagnosis of cannabis use disorder is based on the clinical evaluations of the symptoms and behaviors. However, more objective biomarkers are of interest. To this end, more recently, studies have investigated, if information can be extracted non-invasively from brain activity data. Although these studies have shown promising results, there is still scope for improvement, especially with regards to using more meaningful connectivity analysis approaches [2].

Some evidence has demonstrated that frequent marijuana use affects the connectivity of the brain $[1,2]$. Biomarkers from resting-state functional MRI (rs-fMRI) for frequent marijuana use can be derived using MultiVoxel Pattern Analysis (MVPA) techniques [2,3]. MVPA is a machine-learning framework that extracts differences in patterns of brain connectivity to discriminate between connectivity profiles of individuals with neurological diseases/disorders and healthy individuals. Most MVPA studies commonly use cross-correlation to construct a functional connectivity profile. For example, one such study has obtained an accuracy of 0.69 on the slow frequency bands (0.01-0.1 Hz) [2]. These results demonstrate that meaningful information can be learned from fMRI data. However, correlation does not provide a measure of directed connectivity. Hence, there may be more relevant information in the fMRI data that is not being captured by cross-correlation.

Various methods have been proposed to obtain directional relationships in multivariate time-series data, e.g., transfer entropy [4] and mutual information [5]. However, as the multivariate problem's dimensions increase, computation of the density function becomes computationally expensive [6,7]. Under the Gaussian assumption, transfer entropy is equivalent to Granger causality [8]. However, the computation of multivariate Granger causality for short time series in large-scale problems is challenging $[9,10]$. We introduce large-scale Extended Granger Causality (lsXGC) as a novel method that combines dimension reduction with source time-series augmentation and uses predictive time-series modeling for estimating directed causal relationships among fMRI time-series [11]. In this work, we investigate, if changes in directed connectivity manifest the frequent marijuana use among adults with a childhood diagnosis of ADHD and if such directed measures are better able to discriminate between frequent marijuana users and typical controls. To this end, we apply lsXGC in the MVPA framework for estimating a measure of directed causal interdependence between fMRI time-series. 
This work is embedded in our group's endeavor to expedite artificial intelligence in biomedical imaging by means of advanced pattern recognition and machine learning methods for computational radiology and radiomics, e.g. [ $12-69]$.

\section{DATA}

This data respiratory of the Multimodal Treatment Study of ADHD (MTA) contains a longitudinal study of participants for fourteen years pursued at multiple sites in North America. The study is one of the largest conducted studies for ADHD diagnosis and treatment, with primary results being published in 1999. The Addiction Connectome Preprocessed Initiative (ACPI) data respiratory contains datasets from a subset of MTA participants at six sites with and without childhood ADHD, who were studied as part of a follow-up multimodal brain imaging examination. The principal aim of the MTA study was to investigate the effect of cannabis use among adults with a childhood diagnosis of ADHD. The study was a $2 \times 2$ design of those with and without childhood ADHD and those who did or did not regularly use cannabis [70].

The scan parameters of resting-state fMRI the ACPI dataset can be found on the website of the project [70]. Preprocessed data from the MTA 1 dataset [70] of the ACPI database was used. The dataset included 126 subjects, 101 males and 25 females, with ages between 21-27 years, 86 diagnosed with ADHD (68\%), and 62 of them regularly used marijuana (49\%). Preprocessing of the raw 4D rs-fMRI data had been made using a Configurable Pipeline for the Analysis of Connectomes (C-PAC) [71] and the Advanced Normalization Tools (ANTs) pipeline and consisted in the removal of the first five fMRI volumes, anatomical registration, tissue segmentation, functional registration in the Montreal Neurological Institute (MNI) space, functional masking, temporal bandpass filtering $(0.01-0.1 \mathrm{~Hz})$, motion correction, spatial smoothing, and various nuisance corrections [72]. The MODL parcellation has been used as a pre-computed atlas for regions definition and built using a form of online dictionary learning [2].

\section{METHODS}

\subsection{Large-scale Extended Granger Causality (lsXGC)}

Large-scale Extended Granger Causality (lsXGC) has been developed based on 1) the principle of original Granger causality, which quantifies the causal influence of time-series $\mathbf{x}_{\mathbf{s}}$ on time-series $\mathbf{x}_{\mathbf{t}}$ by quantifying the amount of improvement in the prediction of $\mathbf{x}_{\mathbf{t}}$ in presence of $\mathbf{x}_{\mathbf{s}} .2$ ) the idea of dimension reduction, which resolves the problem of the tackling a under-determined system, which is frequently faced in fMRI analysis, since the number of acquired temporal samples usually is not sufficient for estimating the model parameters $[9,10]$. 
Consider the ensemble of time-series $\mathcal{X} \in \mathbb{R}^{N \times T}$, where $N$ is the number of time-series (Regions Of Interest - ROIs) and $T$ the number of temporal samples. Let $\mathcal{X}=\left(\mathbf{x}_{\mathbf{1}}, \mathbf{x}_{\mathbf{2}}, \ldots, \mathbf{x}_{\mathbf{N}}\right)^{\top}$ be the whole multidimensional system and $x_{i} \in \mathbb{R}^{1 \times T}$ a single time-series with $i=1,2, \ldots, N$, where $\mathbf{x}_{\mathbf{i}}=\left(x_{i}(1), x_{i}(2), \ldots, x_{i}(T)\right)$. In order to overcome the under-determined problem, first $\mathcal{X}$ will be decomposed into its first $p$ high-variance principal components $\mathcal{Z} \in \mathbb{R}^{p \times T}$ using Principal Component Analysis (PCA), i.e.,

$$
\mathcal{Z}=W \mathcal{X}
$$

where $W \in \mathbb{R}^{p \times N}$ represents the PCA coefficient matrix. Subsequently, the dimension-reduced time-series ensemble $\mathcal{Z}$ is augmented by one original time-series $\mathbf{x}_{\mathbf{s}}$ yielding a dimension-reduced augmented time-series ensemble $\mathcal{Y} \in \mathbb{R}^{(p+1) \times T}$ for estimating the influence of $\mathbf{x}_{\mathbf{s}}$ on all other time-series.

Following this, we locally predict $\mathcal{X}$ at each time sample $t$, i.e. $\mathcal{X}(t) \in \mathbb{R}^{N \times 1}$ by calculating an estimate $\hat{\mathcal{X}}_{\mathbf{x}_{\mathbf{s}}}(t)$. To this end, we fit an affine model based on a vector of $m$ vector of $m$ time samples of $\mathcal{Y}(\tau) \in \mathbb{R}^{(p+1) \times 1}(\tau=$ $t-1, t-2, \ldots, t-m)$, which is $\mathbf{y}(t) \in \mathbb{R}^{m \cdot(p+1) \times 1}$, and a parameter matrix $\mathcal{A} \in \mathbb{R}^{N \times m .(p+1)}$ and a constant bias vector $\mathbf{b} \in \mathbb{R}^{N \times 1}$,

$$
\hat{\mathcal{X}}_{\mathbf{x}_{\mathbf{s}}}(t)=\mathcal{A} \mathbf{y}(t)+\mathbf{b}, \quad t=m+1, m+2, \ldots, T .
$$

Now $\hat{\mathcal{X}}_{\mathbf{x}_{\mathbf{s}}}(t)$, which is the prediction of $\mathcal{X}(t)$ without the information of $\mathbf{x}_{\mathbf{s}}$, will be estimated. The estimation processes is identical to the previous one, with the only difference being that we have to remove the augmented time-series $\mathbf{x}_{\mathbf{s}}$ and its corresponding column in the PCA coefficient matrix $W$.

The computation of a lsXGC index is based on comparing the variance of the prediction errors obtained with and without consideration of $\mathbf{x}_{\mathbf{s}}$. The lsXGC index $f_{\mathbf{x}_{\mathbf{s}} \rightarrow \mathbf{x}_{\mathbf{t}}}$, which indicates the influence of $\mathbf{x}_{\mathbf{s}}$ on $\mathbf{x}_{\mathbf{t}}$, can be calculated by the following equation:

$$
f_{\mathbf{x}_{\mathbf{s}} \rightarrow \mathbf{x}_{\mathbf{t}}}=\log \frac{\operatorname{var}\left(e_{s}\right)}{\operatorname{var}\left(e_{\backslash s}\right)}
$$

where $e_{\backslash s}$ is the error in predicting $\mathbf{x}_{\mathbf{t}}$ when $\mathbf{x}_{\mathbf{s}}$ was not considered, and $e_{s}$ is the error, when $\mathbf{x}_{\mathbf{s}}$ was used. In this study, we set $p=8$ and $m=4$.

\subsection{Multi-voxel pattern analysis}

In this study, brain connections served as features for classification and were estimated by two methods, namely lsXGC and cross-correlation. Before using high-dimensional connectivity feature vectors as input to a classifier, feature selection was carried out to reduce the dimension of input features. 


\subsubsection{Feature selection}

To reduce the number of features, feature selection was performed on each training data set with k-fold crossvalidation using Kendall's Tau rank correlation coefficient [73] and 10\%-90\% of test-to-train split ratio. This approach quantifies the relevance of each feature to the task of classification and assigns ranks by testing for independence between different classes for each feature [73].

\subsubsection{Classification}

For the 100 iteration cross-validation scheme for classification, the data set was divided into two groups: a training data set $(90 \%)$ and a test data set $(10 \%)$ in a way that the percentage of samples for each class was preserved. Also, this was repeated with different numbers of features ranging from 5 to 175. A Support Vector Machine (SVM) [74] was used for classification between control subjects and marijuana users. All procedures were implemented using MATLAB 9.8 (MathWorks Inc., Natick, MA, 2020a) and Python 3.8.

\section{RESULTS}

Mean connectivity matrices that were extracted using lsXGC and cross-correlation, are shown in Fig. 1 for both typical control and frequent marijuana user cohorts. Different patterns are visible to the naked eye for both methods. In the following, we quantitatively investigate the difference between connectivity patterns of the two subject cohorts using an MVPA approach.

Figure 1: Mean connectivity matrices: top left: mean connectivity matrix of typical control subjects using lsXGC, top right: mean connectivity matrix of frequent marijuana users using lsXGC, bottom left: mean connectivity matrix of typical controls using cross-correlation, bottom right: mean connectivity matrix of frequent marijuana users using cross-correlation. Note that the different methods appear to extract different connectivity features and that they appear to be slight differences in connectivity patterns between the typical controls and the frequent marijuana users.
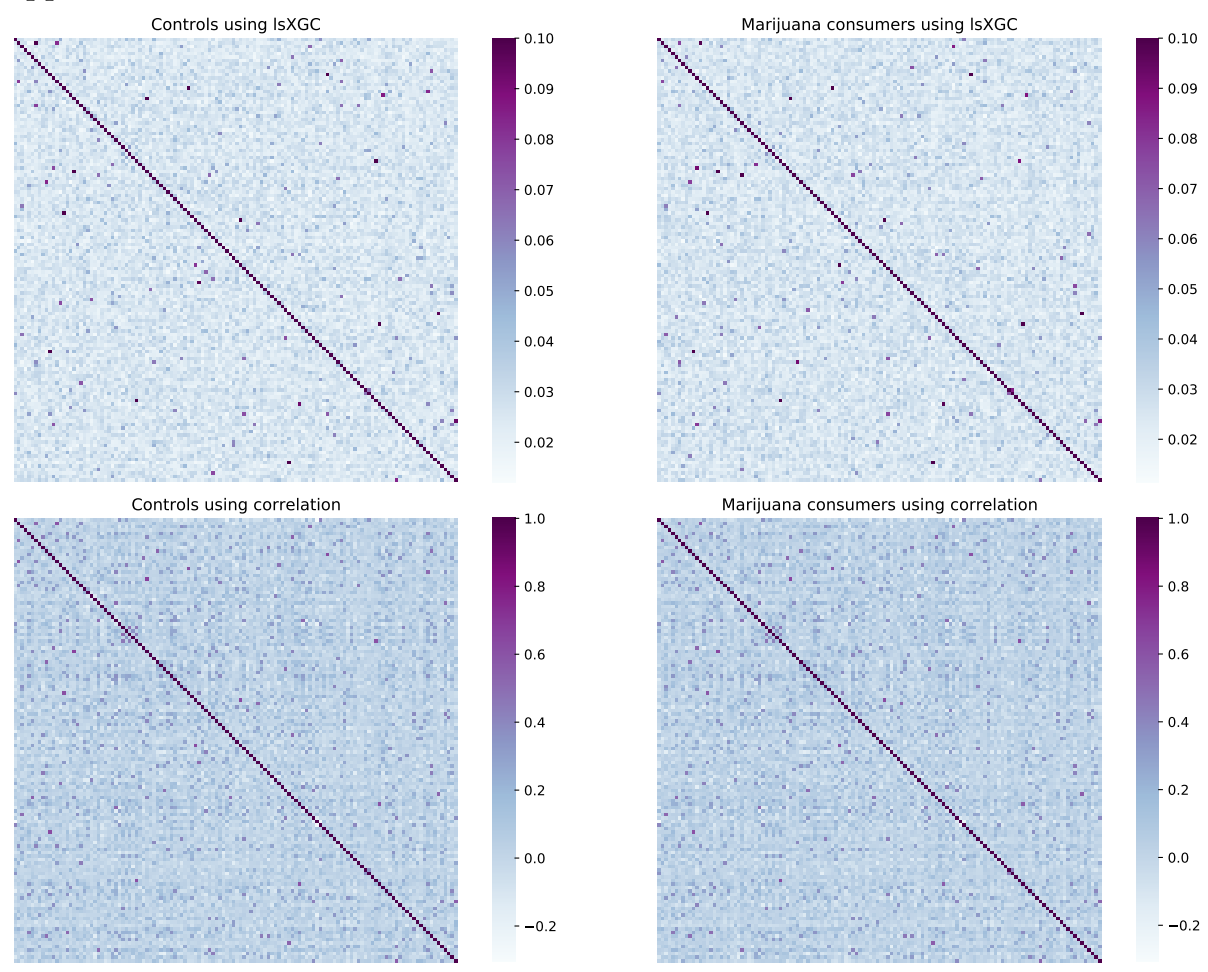




(a) Mean AUC

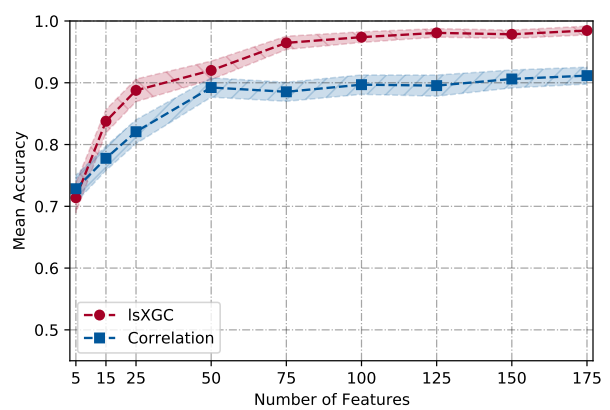

(b) Mean accuracy

Figure 2: Plots comparing the performance of cross-correlation and the proposed large-scale extended Granger causality (lsXGC). The shaded areas represent the 95\% confidence interval. It is clearly demonstrated that lsXGC outperforms cross-correlation for most numbers of selected features.

Classification results were evaluated using the Area Under the Receiver Operator Characteristic Curve (AUC) and accuracy. An $\mathrm{AUC}=1$ indicates a perfect classification, $\mathrm{AUC}=0.5$ indicates random class assignment. In this study, based on preliminary analyses, we chose 8 as the number of the retained components of PCA in the lsXGC algorithm, and model order of 4 for the multivariate vector autoregression function. From the plots of AUC and accuracy results in Fig. 2, we can clearly see that lsXGC outperforms cross-correlation for varying numbers of features. Across the wide range of investigated numbers of features, the performance of lsXGC is consistently good with its mean AUC within $[0.779,0.999]$ and its mean accuracy within $[0.714,0.985]$. On the other hand, cross-correlation performs quite poorly compared to lsXGC with its mean AUC within [0.825, 0.969] and its mean accuracy within $[0.728,0.912]$.

\section{CONCLUSIONS}

In this study, we use a recently developed method for brain connectivity analysis, large-scale Extended Granger Causality (lsXGC), and apply it to subjects of the ACPI data repository in order to classify individuals history of the childhood ADHD diagnosis with frequent marijuana use from typical controls by estimating a measure of directed causal interaction between regional brain activities measured in resting-state fMRI. After constructing connectivity matrices as characterizing features for brain network analysis, we use Kendall's tau rank correlation coefficient for feature selection and a support vector machine for classification. We demonstrate that lsXGC compares favorably to conventional cross-correlation analysis, as indicated by the significantly higher AUC and accuracy values. The potential of lsXGC as an effective biomarker for identifying frequent marijuana use is yet to be validated in prospective clinical trials. However, our results suggest that our approach outperforms the current clinical standard, namely cross-correlation, at uncovering meaningful information from functional MRI data. 


\section{ACKNOWLEDGMENTS}

This research was funded by Ernest J. Del Monte Institute for Neuroscience Award from the Harry T. Mangurian Jr. Foundation. This work was conducted as a Practice Quality Improvement (PQI) project related to American Board of Radiology (ABR) Maintenance of Certificate (MOC) for Prof. Dr. Axel Wismüller. This work is not being and has not been submitted for publication or presentation elsewhere.

\section{REFERENCES}

[1] Solowij, N., [Cannabis and cognitive functioning], Cambridge University Press (2006).

[2] Dadi, K., Rahim, M., Abraham, A., Chyzhyk, D., Milham, M., Thirion, B., Varoquaux, G., Initiative, A. D. N., et al., "Benchmarking functional connectome-based predictive models for resting-state fMRI," Neuroimage 192, 115-134 (2019).

[3] Norman, K. A., Polyn, S. M., Detre, G. J., and Haxby, J. V., "Beyond mind-reading: multi-voxel pattern analysis of fMRI data," Trends in cognitive sciences 10(9), 424-430 (2006).

[4] Schreiber, T., "Measuring information transfer," Physical review letters 85(2), 461 (2000).

[5] Kraskov, A., Stögbauer, H., and Grassberger, P., "Estimating mutual information," Physical review E 69(6), 066138 (2004).

[6] Mozaffari, M. and Yilmaz, Y., "Online multivariate anomaly detection and localization for high-dimensional settings," arXiv preprint arXiv:1905.07107 (2019).

[7] Mozaffari, M. and Yilmaz, Y., "Online anomaly detection in multivariate settings," in [2019 IEEE 29th International Workshop on Machine Learning for Signal Processing (MLSP)], 1-6, IEEE (2019).

[8] Barnett, L., Barrett, A. B., and Seth, A. K., "Granger causality and transfer entropy are equivalent for Gaussian variables," Physical review letters 103(23), 238701 (2009).

[9] Vosoughi, M. A. and Wismüller, A., "Large-scale kernelized Granger causality to infer topology of directed graphs with applications to brain networks," arXiv preprint arXiv:2011.08261 (2020).

[10] Wismüller, A., DSouza, A. M., Abidin, A. Z., and Vosoughi, M. A., "Large-scale nonlinear Granger causality: A data-driven, multivariate approach to recovering directed networks from short time-series data," arXiv preprint arXiv:2009.04681 (2020).

[11] Wismüller, A., Foxe, J. J., Geha, P., and Saboksayr, S. S., "Large-scale extended Granger causality (lsxgc) for classification of autism spectrum disorder from resting-state functional MRI," in [Medical Imaging 2020: Computer-Aided Diagnosis], 11314, 113141Y, International Society for Optics and Photonics (2020).

[12] Nattkemper, T. W. and Wismüller, A., "Tumor feature visualization with unsupervised learning," Medical Image Analysis 9(4), 344-351 (2005). 
[13] Bunte, K., Hammer, B., Wismüller, A., and Biehl, M., "Adaptive local dissimilarity measures for discriminative dimension reduction of labeled data," Neurocomputing 73(7-9), 1074-1092 (2010).

[14] Wismueller, A., Vietze, F., and Dersch, D. R., "Segmentation with neural networks," in [Handbook of medical imaging], 107-126, Academic Press, Inc. (2000).

[15] Leinsinger, G., Schlossbauer, T., Scherr, M., Lange, O., Reiser, M., and Wismüller, A., "Cluster analysis of signal-intensity time course in dynamic breast MRI: does unsupervised vector quantization help to evaluate small mammographic lesions?," European radiology 16(5), 1138-1146 (2006).

[16] Wismüller, A., Vietze, F., Behrends, J., Meyer-Baese, A., Reiser, M., and Ritter, H., "Fully automated biomedical image segmentation by self-organized model adaptation," Neural Networks 17(8-9), 1327-1344 (2004).

[17] Hoole, P., Wismüller, A., Leinsinger, G., Kroos, C., Geumann, A., and Inoue, M., "Analysis of tongue configuration in multi-speaker, multi-volume MRI data," (2000).

[18] Wismüller, A., "Exploratory morphogenesis (xom): a novel computational framework for self-organization," Ph. D. thesis, Technical University of Munich, Department of Electrical and Computer Engineering (2006).

[19] Wismüller, A., Dersch, D. R., Lipinski, B., Hahn, K., and Auer, D., "A neural network approach to functional MRI pattern analysis - clustering of time-series by hierarchical vector quantization," in [International Conference on Artificial Neural Networks], 857-862, Springer (1998).

[20] Wismüller, A., Vietze, F., Dersch, D. R., Behrends, J., Hahn, K., and Ritter, H., "The deformable feature map-a novel neurocomputing algorithm for adaptive plasticity in pattern analysis," Neurocomputing 48(1-4), 107-139 (2002).

[21] Behrends, J., Hoole, P., Leinsinger, G. L., Tillmann, H. G., Hahn, K., Reiser, M., and Wismüller, A., "A segmentation and analysis method for MRI data of the human vocal tract," in [Bildverarbeitung für die Medizin 2003], 186-190, Springer (2003).

[22] Wismuller, A., "Neural network computation in biomedical research: chances for conceptual crossfertilization," Theory in Biosciences (1997).

[23] Bunte, K., Hammer, B., Villmann, T., Biehl, M., and Wismüller, A., "Exploratory observation machine (xom) with kullback-leibler divergence for dimensionality reduction and visualization.," in $[E S A N N], \mathbf{1 0}$, 87-92 (2010).

[24] Wismüller, A., Vietze, F., Dersch, D. R., Hahn, K., and Ritter, H., "The deformable feature map-adaptive plasticity for function approximation," in [International Conference on Artificial Neural Networks], 123-128, Springer (1998). 
[25] Wismüller, A., "The exploration machine-a novel method for data visualization," in [International Workshop on Self-Organizing Maps], 344-352, Springer (2009).

[26] Wismüller, A., "Method, data processing device and computer program product for processing data," (July 28 2009). US Patent 7,567,889.

[27] Huber, M. B., Nagarajan, M., Leinsinger, G., Ray, L. A., and Wismüller, A., "Classification of interstitial lung disease patterns with topological texture features," in [Medical Imaging 2010: Computer-Aided Diagnosis], 7624, 762410, International Society for Optics and Photonics (2010).

[28] Wismüller, A., "The exploration machine: a novel method for analyzing high-dimensional data in computeraided diagnosis," in [Medical Imaging 2009: Computer-Aided Diagnosis], 7260, 72600G, International Society for Optics and Photonics (2009).

[29] Bunte, K., Hammer, B., Villmann, T., Biehl, M., and Wismüller, A., "Neighbor embedding xom for dimension reduction and visualization," Neurocomputing 74(9), 1340-1350 (2011).

[30] Meyer-Bäse, A., Lange, O., Wismüller, A., and Ritter, H., "Model-free functional MRI analysis using topographic independent component analysis," International journal of neural systems 14(04), 217-228 (2004).

[31] Wismüller, A., "A computational framework for nonlinear dimensionality reduction and clustering," in [International Workshop on Self-Organizing Maps], 334-343, Springer (2009).

[32] Meyer-Base, A., Auer, D., and Wismueller, A., "Topographic independent component analysis for fMRI signal detection," in [Proceedings of the International Joint Conference on Neural Networks, 2003.], 1, 601-605, IEEE (2003).

[33] Meyer-Baese, A., Schlossbauer, T., Lange, O., and Wismüller, A., "Small lesions evaluation based on unsupervised cluster analysis of signal-intensity time courses in dynamic breast MRI," International journal of biomedical imaging 2009 (2009).

[34] Wismueller, A., Lange, O., Auer, D., and Leinsinger, G., "Model-free functional MRI analysis for detecting low-frequency functional connectivity in the human brain," in [Medical Imaging 2010: Computer-Aided Diagnosis], 7624, 76241M, International Society for Optics and Photonics (2010).

[35] Meyer-Bäse, A., Saalbach, A., Lange, O., and Wismüller, A., "Unsupervised clustering of fMRI and MRI time series," Biomedical Signal Processing and Control 2(4), 295-310 (2007).

[36] Huber, M. B., Nagarajan, M. B., Leinsinger, G., Eibel, R., Ray, L. A., and Wismüller, A., "Performance of topological texture features to classify fibrotic interstitial lung disease patterns," Medical Physics 38(4), 2035-2044 (2011). 
[37] Wismüller, A., Verleysen, M., Aupetit, M., and Lee, J. A., "Recent advances in nonlinear dimensionality reduction, manifold and topological learning.," in [ESANN], (2010).

[38] Meyer-Baese, A., Lange, O., Wismüller, A., and Hurdal, M. K., "Analysis of dynamic susceptibility contrast MRI time series based on unsupervised clustering methods," IEEE Transactions on Information Technology in Biomedicine 11(5), 563-573 (2007).

[39] Wismueller, A., Behrends, J., Hoole, P., Leinsinger, G. L., Reiser, M. F., and Westesson, P.-L., "Human vocal tract analysis by in vivo 3d MRI during phonation: a complete system for imaging, quantitative modeling, and speech synthesis," in [International Conference on Medical Image Computing and ComputerAssisted Intervention], 306-312, Springer (2008).

[40] Wismüller, A., "Method and device for representing multichannel image data," (Nov. 17 2015). US Patent $9,189,846$.

[41] Huber, M. B., Bunte, K., Nagarajan, M. B., Biehl, M., Ray, L. A., and Wismüller, A., "Texture feature ranking with relevance learning to classify interstitial lung disease patterns," Artificial intelligence in medicine 56(2), 91-97 (2012).

[42] Wismuller, A., Meyer-Baese, A., Lange, O., Reiser, M. F., and Leinsinger, G., "Cluster analysis of dynamic cerebral contrast-enhanced perfusion MRI time-series," IEEE transactions on medical imaging 25(1), 62-73 (2005).

[43] Twellmann, T., Saalbach, A., Muller, C., Nattkemper, T. W., and Wismuller, A., "Detection of suspicious lesions in dynamic contrast enhanced MRI data," in [The 26th Annual International Conference of the IEEE Engineering in Medicine and Biology Society], 1, 454-457, IEEE (2004).

[44] Otto, T. D., Meyer-Baese, A., Hurdal, M., Sumners, D., Auer, D., and Wismuller, A., "Model-free functional MRI analysis using cluster-based methods," in [Intelligent Computing: Theory and Applications], 5103, 1724, International Society for Optics and Photonics (2003).

[45] Varini, C., Nattkemper, T. W., Degenhard, A., and Wismuller, A., "Breast MRI data analysis by lle," in [2004 IEEE International Joint Conference on Neural Networks (IEEE Cat. No. 04CH37541)], 3, 24492454, IEEE (2004).

[46] Huber, M. B., Lancianese, S. L., Nagarajan, M. B., Ikpot, I. Z., Lerner, A. L., and Wismuller, A., "Prediction of biomechanical properties of trabecular bone in $\mathrm{mr}$ images with geometric features and support vector regression," IEEE Transactions on Biomedical Engineering 58(6), 1820-1826 (2011).

[47] Meyer-Base, A., Pilyugin, S. S., and Wismuller, A., "Stability analysis of a self-organizing neural network with feedforward and feedback dynamics," in [2004 IEEE International Joint Conference on Neural Networks (IEEE Cat. No. 04CH37541)], 2, 1505-1509, IEEE (2004). 
[48] Meyer-Baese, A., Lange, O., Schlossbauer, T., and Wismuller, A., "Computer-aided diagnosis and visualization based on clustering and independent component analysis for breast MRI," in [2008 15th IEEE International Conference on Image Processing], 3000-3003, IEEE (2008).

[49] Wismüller, A., Meyer-Bäse, A., Lange, O., Schlossbauer, T., Kallergi, M., Reiser, M., and Leinsinger, G., "Segmentation and classification of dynamic breast magnetic resonance image data," Journal of Electronic Imaging 15(1), 013020 (2006).

[50] Bhole, C., Pal, C., Rim, D., and Wismüller, A., "3d segmentation of abdominal ct imagery with graphical models, conditional random fields and learning," Machine vision and applications 25(2), 301-325 (2014).

[51] Nagarajan, M. B., Coan, P., Huber, M. B., Diemoz, P. C., Glaser, C., and Wismüller, A., "Computer-aided diagnosis in phase contrast imaging x-ray computed tomography for quantitative characterization of ex vivo human patellar cartilage," IEEE Transactions on Biomedical Engineering 60(10), 2896-2903 (2013).

[52] Wismüller, A., Meyer-Bäse, A., Lange, O., Auer, D., Reiser, M. F., and Sumners, D., "Model-free functional MRI analysis based on unsupervised clustering," Journal of Biomedical Informatics 37(1), 10-18 (2004).

[53] Meyer-Baese, A., Wismueller, A., Lange, O., and Leinsinger, G., "Computer-aided diagnosis in breast MRI based on unsupervised clustering techniques," in [Intelligent Computing: Theory and Applications II], 5421, 29-37, International Society for Optics and Photonics (2004).

[54] Nagarajan, M. B., Coan, P., Huber, M. B., Diemoz, P. C., Glaser, C., and Wismüller, A., "Computer-aided diagnosis for phase-contrast x-ray computed tomography: quantitative characterization of human patellar cartilage with high-dimensional geometric features," Journal of digital imaging 27(1), 98-107 (2014).

[55] Nagarajan, M. B., Huber, M. B., Schlossbauer, T., Leinsinger, G., Krol, A., and Wismüller, A., "Classification of small lesions on dynamic breast MRI: Integrating dimension reduction and out-of-sample extension into cadx methodology," Artificial intelligence in medicine 60(1), 65-77 (2014).

[56] Yang, C.-C., Nagarajan, M. B., Huber, M. B., Carballido-Gamio, J., Bauer, J. S., Baum, T. H., Eckstein, F., Lochmüller, E.-M., Majumdar, S., Link, T. M., et al., "Improving bone strength prediction in human proximal femur specimens through geometrical characterization of trabecular bone microarchitecture and support vector regression," Journal of electronic imaging 23(1), 013013 (2014).

[57] Wismüller, A., Nagarajan, M. B., Witte, H., Pester, B., and Leistritz, L., "Pair-wise clustering of large scale Granger causality index matrices for revealing communities," in [Medical Imaging 2014: Biomedical Applications in Molecular, Structural, and Functional Imaging], 9038, 90381R, International Society for Optics and Photonics (2014).

[58] Wismüller, A., Wang, X., DSouza, A. M., and Nagarajan, M. B., "A framework for exploring non-linear functional connectivity and causality in the human brain: mutual connectivity analysis (mca) of resting-state 
functional MRI with convergent cross-mapping and non-metric clustering," arXiv preprint arXiv:1407.3809 (2014).

[59] Schmidt, C., Pester, B., Nagarajan, M., Witte, H., Leistritz, L., and Wismueller, A., "Impact of multivariate Granger causality analyses with embedded dimension reduction on network modules," in [2014 36th Annual International Conference of the IEEE Engineering in Medicine and Biology Society], 2797-2800, IEEE (2014).

[60] Wismüller, A., Abidin, A. Z., D’Souza, A. M., Wang, X., Hobbs, S. K., Leistritz, L., and Nagarajan, M. B., "Nonlinear functional connectivity network recovery in the human brain with mutual connectivity analysis (MCA): convergent cross-mapping and non-metric clustering," in [Medical Imaging 2015: Biomedical Applications in Molecular, Structural, and Functional Imaging], 9417, 94170M, International Society for Optics and Photonics (2015).

[61] Wismüller, A., Abidin, A. Z., DSouza, A. M., and Nagarajan, M. B., "Mutual connectivity analysis (MCA) for nonlinear functional connectivity network recovery in the human brain using convergent cross-mapping and non-metric clustering," in [Advances in Self-Organizing Maps and Learning Vector Quantization], 217226, Springer (2016).

[62] Schmidt, C., Pester, B., Schmid-Hertel, N., Witte, H., Wismüller, A., and Leistritz, L., "A multivariate Granger causality concept towards full brain functional connectivity," PloS one 11(4) (2016).

[63] Abidin, A. Z., Chockanathan, U., DSouza, A. M., Inglese, M., and Wismüller, A., "Using large-scale granger causality to study changes in brain network properties in the clinically isolated syndrome (CIS) stage of multiple sclerosis," in [Medical Imaging 2017: Biomedical Applications in Molecular, Structural, and Functional Imaging], 10137, 101371B, International Society for Optics and Photonics (2017).

[64] DSouza, A. M., Abidin, A. Z., Leistritz, L., and Wismüller, A., "Exploring connectivity with large-scale Granger causality on resting-state functional MRI," Journal of neuroscience methods 287, 68-79 (2017).

[65] Chen, L., Wu, Y., DSouza, A. M., Abidin, A. Z., Wismüller, A., and Xu, C., "MRI tumor segmentation with densely connected 3d cnn," in [Medical Imaging 2018: Image Processing], 10574, 105741F, International Society for Optics and Photonics (2018).

[66] Abidin, A. Z., DSouza, A. M., Nagarajan, M. B., Wang, L., Qiu, X., Schifitto, G., and Wismüller, A., "Alteration of brain network topology in hiv-associated neurocognitive disorder: A novel functional connectivity perspective," NeuroImage: Clinical 17, 768-777 (2018).

[67] Abidin, A. Z., Deng, B., DSouza, A. M., Nagarajan, M. B., Coan, P., and Wismüller, A., "Deep transfer learning for characterizing chondrocyte patterns in phase contrast x-ray computed tomography images of the human patellar cartilage," Computers in biology and medicine 95, 24-33 (2018). 
[68] DSouza, A. M., Abidin, A. Z., Chockanathan, U., Schifitto, G., and Wismüller, A., "Mutual connectivity analysis of resting-state functional MRI data with local models," NeuroImage 178, 210-223 (2018).

[69] Chockanathan, U., DSouza, A. M., Abidin, A. Z., Schifitto, G., and Wismüller, A., "Automated diagnosis of HIV-associated neurocognitive disorders using large-scale Granger causality analysis of resting-state functional MRI," Computers in Biology and Medicine 106, 24-30 (2019).

[70] MTA 1, "http://fcon_1000.projects.nitrc.org/indi/acpi/html/," (2015). Last accessed 20 August 2020.

[71] C-PAC, "https://fcp-indi.github.io/," (2015). Last accessed 20 August 2020.

[72] The INDI Team, "ACPI preprocessing pipelines," (2015). Last accessed 20 August 2020.

[73] Kendall, M. G., "The treatment of ties in ranking problems," Biometrika 33(3), 239-251 (1945).

[74] Suykens, J. A. and Vandewalle, J., "Least squares support vector machine classifiers," Neural processing letters $\mathbf{9}(3)$, 293-300 (1999). 